\title{
Surface Modification of Implants by Sol-Gel Coating Technology: Advantages and Applications
}

\author{
Flavia Bollino ${ }^{1}$ and Elisabetta Tranquillo ${ }^{2}$ \\ 1 University of Campania “Luigi Vanvitelli", Department of Engineering \\ 2 University of Manchester, Department of Mechanical, Aerospace and Civil Engineering \\ * Correspondence: flavia.bollino@unicampania.it (F.B.); elisabetta.tranquillo@unicampania.it (E.T.)
}

Received: 17 April 2020; Accepted: 8 May 2020; Published: 12 May 2020

\begin{abstract}
Nowadays, approximately 1.5 million joint replacements are performed annually in Europe, while 7 million in the United States. Despite the advances made in the biomaterials field over the last 50 years, still today the average lifetime of an implant is about 20 years. his entails the need for subsequent prosthetic device replacement, especially in young patients, resulting in an increase in patients' health risks as well as clinical and economic burdens for the public health service.

The failure of the implants can be caused by several reasons, such as adverse immune system reaction, biofilm formation or mechanical, chemical, tribological, surgical, manufacturing and biocompatibility problems. An alternative and useful strategy used to overcome this limitation is the modification of the implants' surface by sol-gel coating technology. It allows the production of coatings with a wide range of properties on substrates of different nature and shape, due to the fine control of the coating composition and microstructure.

Sol-gel coatings were successfully proposed to inhibit wear, reduce corrosion and ion release, modify lubricity, hydrophilicity/hydrophobicity, and functionality of several substrates. Moreover, many works report the application of sol-gel coatings on bio-inert implants to improve their bioactivity and biocompatibility, leading to the enhancement of the integration process. This is ascribable to the presence of residual hydroxyl groups on coating materials' surface, able to induce easier nucleation of the hydroxyapatite, to their mesoporosity and, thus, the large specific surface area. Further, the low processing temperatures allows easy coating functionalization by embedding suitable molecules such as anti-inflammatory and antibacterial agents leading to coatings preventing biofilm formation and inflammatory pathway activation.

Therefore, the application of the sol-gel coatings provides an excellent chemical modification of the materials' surface allowing protective barrier layers production.
\end{abstract}

\section{References}

Xiao-Yu Yang, Nidhi Chauhan. Photoenergy and Thin Film Materials, Advanced Materials Series. Chapter 8. WILEY-Scrivener, USA.

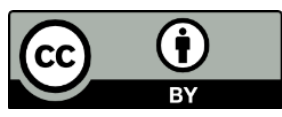

(C) 2020 by the authors. Submitted for possible open access publication under the terms and conditions of the Creative Commons Attribution (CC BY) license (http://creativecommons.org/licenses/by/4.0/). 